\title{
MÉTODOS DE PROTECCIÓN EN FAJA Y PROTECCIÓN UNIFORME EN EL ÁREA DE NELTUME Y COÑARIPE
}

\section{LUIS OTERO D.(*), LUIS BARRALES M.(**), ALVARO CONTRERAS( $(* *)$ e IGNACIO OJEDA H. $(* * *)$}

\author{
(*) Ing. Forestal, M.Sc. Medio Ambiente \\ (**) Técnico Forestal \\ $(* *)$ Ing. Forestal (E) \\ $(* * *)$ Ing. Forestal (E) \\ Instituto Forestal-Valdivia
}

\section{RESUMEN}

Este articulo presenta los resultados obtenidos en plantaciones de rauli (Nothofagus alpina) realizadas bajo el método de Protección en Fajas y Protección Uniforme. Los resultados están referidos al crecimiento inicial de plantaciones durante las primeras dos temporadas de crecimiento.

Los ensayos se realizaron en predios de la empresa BOMASA, en los fundos Pilmaiquén. zona de Neltume, y Quilalelfu, área de Coñaripe. Los ensayos se encuentran cerca del limite altitudinal de la especie, en los 800 y 1200 msnm, respectivamente, por lo tanto en condiciones climáticas relativamente rigurosas.

Las plantaciones se realizaron en fajas de $45 \mathrm{~m}$ de ancho y $60 \mathrm{~m}$ de ancho (1.5 a 2 veces la altura del dosel protector) y bajo el método de Protección Uniforme con una cobertura de 30\%.

Los resultados generales indican que el crecimiento medio anual en altura de las plantaciones, bajo el método de Protección en Fajas, es de $42 \mathrm{~cm}$ y llega hasta $57 \mathrm{~cm}$ para plantas fertilizadas, durante la segunda temporada de crecimiento. Lo cual es relativamente alto si se compara con otros estudios realizados en plantaciones con esta especie. En el caso de la Protección Uniforme el incremento en altura es de $34 \mathrm{~cm}$ anuales.

En el sistema de cortas en fajas el prendimiento medio es de $86 \%$ y el mismo porcentaje fue determinado en términos de plantas calificadas como de buen vigor. Los porcentajes de plantas con doble flecha fluctuaron entre el $2 \%$ y el $19 \%$.

Se encontraron diferencias significativas entre parcelas fertilizadas con abono foliar y otras testigos. Las diferencias de crecimiento medio entre el tratamiento y el control fue de $16 \mathrm{~cm}$ el último año.

Tambiè se encontraron diferencias significativas entre los crecimientos en altura para plantas ubicadas a diferentes distancias a la protección, siendo los crecimientos un 30 a $40 \%$ inferiores para las parcelas ubicadas en la parte alta de la faja, vale decir en condiciones de menor protección lateral, dada la exposición norte de la ladera donde se efectuó la corta.

Los resultados obtenidos demuestran y confirman el vigor y potencial productivo de esta especie, la cual puede ser plantada con éxito con protección lateral, respondiendo bien a situaciones de alta luminosidad. 


\section{ABSTRACT}

This study presents the results obtained in a Rauli plantation (Nothofagus alpina) established under strip cut and shelterbelt system. This study includes the first two seasons of growth. The trials are established in the field of the forest company (BOMASA S.A.) in the Panguipulli area $\left(40^{\circ} \mathrm{LS}\right.$ and $71^{\circ} 50 \mathrm{LW}$ ), located between $800-1200 \mathrm{~m}$ above sea level, wich is an upper elevation level for a Rauli forest.

The strip cut system consist of cut areas where the width of the strip cut is equivalent to 1.5 times and 2 times the height of the dominant canopv. The strip cuts follow the grade level and altemate with an untouched forest strip of $40 \mathrm{~m}$ in width.

The results show that the annual growth of the plantation in the strip system is greater than the sheltenwood system. The mean annual growth in height during the second season of growth in the strip cut of 1.5 times the canopy height is $42 \mathrm{~cm}$. In contrast, the shelterwood system has a mean annual growth in height of $34 \mathrm{~cm}$. In the strip cup of 2 times the canopy height, the mean growth is $37 \mathrm{~cm} / \mathrm{yr}$.

In both strip cut widths the growth varies $30 \%-40 \%$ between the boarder and the central part of the strip cut. In addition, it was observed that the growth was notably better near de boarder of the strip cut.

Keywords: Silviculture, Nothofagus alpina 


\section{INTRODUCCIÓN}

Las nuevas tendencias en silvicultura de bosque nativo se orientan hacia intervenciones más intensivas, caracterizadas por una extracción alta del volumen por hectárea, bajo distintas modalidades del Método de Protección, ya sea protección lateral ( en fajas o claros de bosque) o protección vertical (uniforme). Estudios ambientales realizados en estos sistemas de corta demuestra sus ventajas y los efectos de la protección tanto vertical como lateral (Otero et al, 1994 a y b). Los resultados demuestran la existencia de protección lateral en fajas de corta de hasta dos veces la altura del dosel y en cortas de protección uniforme con extracciones del $70 \%$ de la cobertura.

En este esquema de una silvicultura intensiva, la plantación constituye una herramienta fundamental del manejo. Evaluaciones realizadas en el ex Complejo Panguipulli revelan significativos crecimientos para todas las especies de Nothofagus que han sido plantadas (Donoso et al,1993). Los resultados de este estudio muestran incrementos medios anuales en diámetros de $1,2 \mathrm{~cm}$, con crecimientos periódicos de hasta $1,5 \mathrm{~cm}$ anuales. Las estimaciones de volumen para plantaciones jóvenes, de 15 años, revelan crecimientos de 12 a $17 \mathrm{~m}^{3} / \mathrm{ha} / \mathrm{año}$.

Otro estudio realizado en una plantación de 15 años del ex Complejo Panguipulli, entrega un crecimiento medio anual en diámetro de $0,88 \mathrm{~cm}$ y un crecimiento anual periódico máximo de $1,36 \mathrm{~cm}$ a los 10 años. El crecimiento medio anual en altura es de $0,9 \mathrm{~m}$. La proyección de este rodal a las actuales tasa de crecimientos, entrega un volumen de $658 \mathrm{~m}^{3}$ a los 35 años, vale decir un crecimiento anual de $18.8 \mathrm{~m}^{3} / \mathrm{ha} / \mathrm{año}$ (Contreras et al,1995).

De igual forma los resultados en Gran Bretaña, con ensayos de cerca de 35 años, confirman el potencial productivo de esta especie. Plantaciones de 35 años alcanzan un DAP de $53 \mathrm{~cm}$, en los mejores sitios, sin heladas, y en promedio los crecimientos son de alrededor de $1,2 \mathrm{~cm}$ anuales en los ensayos establecidos en sitios cerca de la costa. (Danby, 1991).

En plantaciones de raulí, de 37 años en Inglaterra, se han obtenido crecimientos del orden de los $17,2 \mathrm{~m}^{3} / \mathrm{ha} / \mathrm{año}$, con volúmenes acumulados de $636 \mathrm{~m}^{3} / \mathrm{ha}$. Para sitios de menor calidad, con más heladas, los crecimientos son de $14 \mathrm{~m}^{3} / \mathrm{ha} /$ año (Tuley, 1979).

Es de suponer que con semilla seleccionada y con la nueva tecnologia de viverización que existe y que se aplica al cultivo de especies exóticas, los crecimientos podrian ser aún mayores. 
Diversas investigaciones revelan el alto requerimiento de luminosidad de la especie, adaptándose bien a condiciones de campo abierto como las presentadas bajo el sistema de protección en fajas.

Otros estudios en bosques diferentes, como los bosques del Tipo Siempreverde, revelan que los crecimientos de la regeneración establecida post cosecha son también superiores bajo el sistema de Protección en Fajas que bajo otros métodos silviculturales (Donoso.1989).

Experiencias realizadas en bosques tropicales en Penú y Costa Rica muestran también que el método de las fajas proporciona buenas condiciones para la regeneración natural del bosque y presenta importantes ventajas ecológicas (OcañaVidal. 1992: Dykstra y Heinrich. 1992).

\section{ÁREA DE ESTUdIO}

El estudio se llevó a cabo en los predios Pilmaiquén y Quilalelfu, propiedad de la empresa BOMASA S.A.

El fundo Pilmaiquén esta ubicado en la Cordillera de los Andes. Provincia de Valdivia, en el área de Neltume $\left(71^{\circ} 50^{\prime} \mathrm{LW}\right.$ y $\left.40^{\circ} 00^{\prime} \mathrm{LS}\right)$. La altitud del área fluctúa entre los 870 y 1050 msnm.

El fundo Quilalelfu se encuentra en el área de Coñaripe ( $71^{\circ} 50^{\prime} \mathrm{LW}$ y $\left.39^{\circ} 34^{\prime} \mathrm{LS}\right)$ a una altitud de $1100 \mathrm{msnm}$.

La exposición para el área de Pilmaiquén es norte, con pendientes de 35 a $40 \%$. En Quilalelfu la pendiente es inferior al $15 \%$.

El clima de la zona es de montaña, caracterizado por elevadas precipitaciones. Aproximadamente $4400 \mathrm{~mm}$ anuales, con un mínimo de $180 \mathrm{~mm}$ en el mes de enero, $669 \mathrm{~mm}$ en el mes de junio y una importante cantidad en forma de nieve.

La temperatura media anual es de $11.3^{\circ} \mathrm{C}$. En verano sube hasta $16^{\circ} \mathrm{C}$, con extremas que superan los $30^{\circ} \mathrm{C}$ (Otero et al .1995). En invierno el promedio baja a $6.5^{\circ}$ $\mathrm{C}$ con extremas de $-9^{\circ} \mathrm{C}$.

Los suelos corresponden a trumaos desarrollados sobre arena y escoria volcánica de diversa granulometría. Su textura es franco arenosa. de alta permeabilidad, poseen un alto poder fijador de fosfatos y baja densidad aparente (Peralta y Oyanedel, 1980).

La vegetación del área corresponde al tipo forestal Coihue-Rauli-Tepa de Altura, en el cual la tepa ha sido reemplazada por lenga. Se trata de un bosque en estado maduro, con grandes árboles y escasa regeneración. Estos bosques han sido floreados, particularmente el rauli. 


\section{MATERIAL Y MÉTODO}

\section{Diseño}

El ensayo considera aproximadamente 1,1 hectáreas de plantaciones de raulí bajo el sistema de Protección en Fajas y 1 ha bajo Protección Uniforme.

En la Protección Uniforme se dividió la hectárea plantada en 25 parcelas de $20 \mathrm{~m}$ por $20 \mathrm{~m}$, de estas se sortearon 9 las cuales se utilizaron en la caracterización (Figura $\left.\mathrm{N}^{\circ} 1\right)$.

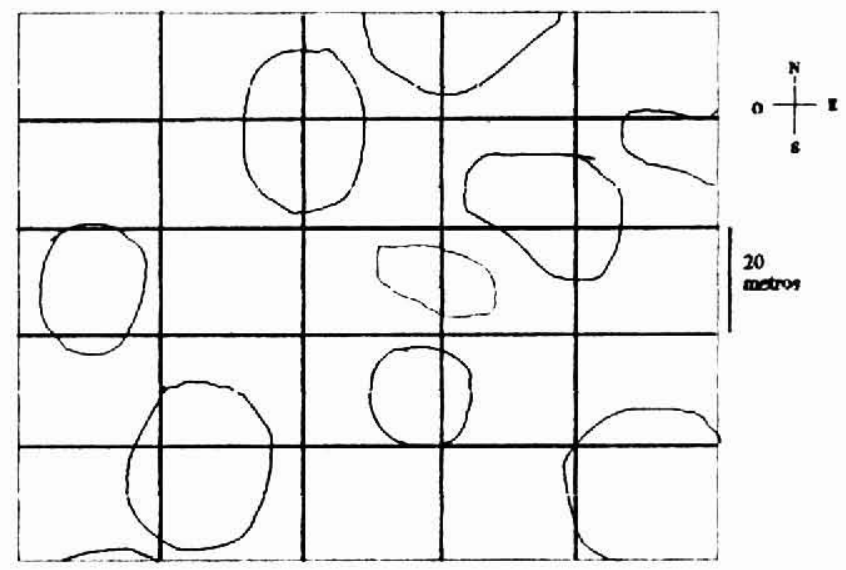

Figura № 1. PROYECCIÓN HORIZONTAL MÉTODO PROTECCIÓN UNIFORME.

En cada faja se marcaron tres bloques o tratamientos, en su límite superior, al medio y en el limite inferior de cada faja. Cada bloque fue considerado como un tratamiento distinto debido al efecto de protección de las fajas y está compuesto de 3 parcelas escogidas al azar de entre 6 , con un promedio de 25 plantas cada una, (Figuras $\mathrm{N}^{\mathrm{os}} 2$ y 3). 


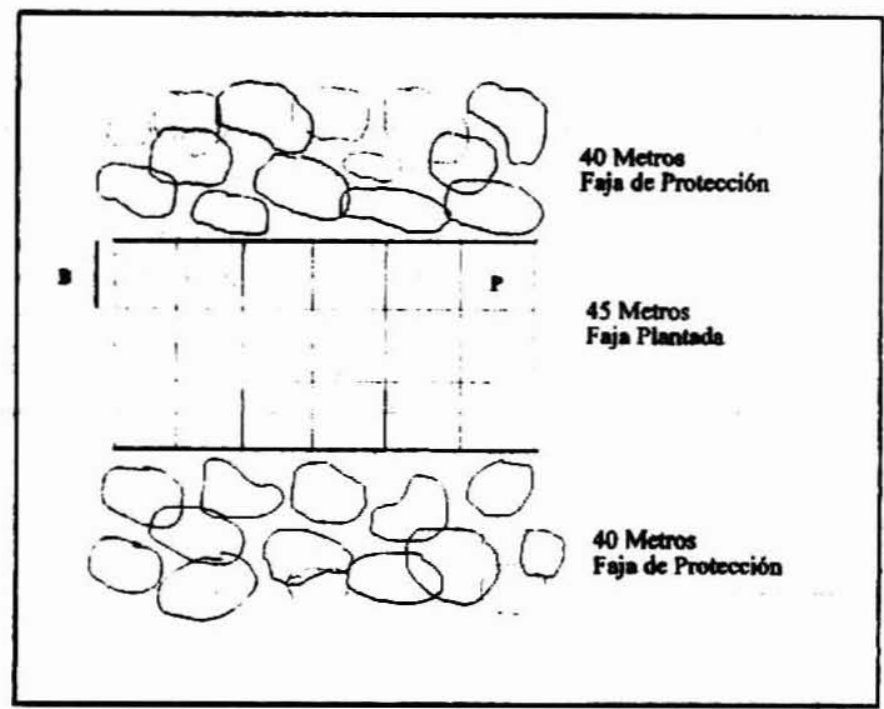

Figura N $N^{\circ}$ 2. PROYECCIÓN HORIZONTAL PROTECCIÓN EN FAJA (45 m). B: BLOQUE, P: PARCELA.

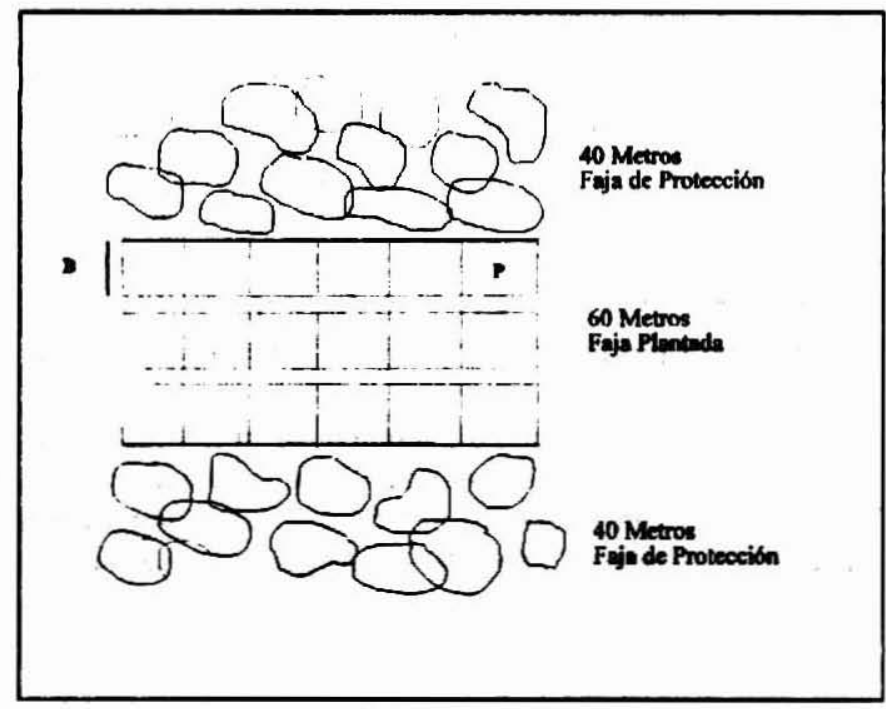

Figura $\mathrm{N}^{\circ}$ 3. PROYECCIÓN HORIZONTAL PROTECCIÓN FAJA $(60 \mathrm{~m})$ B: BLOQUE, P: PARCELA. 
Cada planta fue individualizada con un anillo plástico y con una lámina de cobre para asegurar su identificación posterior.

El segundo tratamiento considerado es la fertilización con abono foliar (Bayfolan), con posterioridad a la plantación de 1992.

Las variables consideradas en el estudio son:

- Altura $(\mathrm{H})$ y diámetro de cuello (DAC).

- Sobrevivencia.

- Vigor. Medido en 3 categorias: (1) vigorosas, (2) buen estado, (3) plantas debilitadas.

- Forma : (1) = una flecha; (2) = doble flecha (dos o más flechas).

Las plantaciones realizadas en la Faja de $60 \mathrm{~m}$ y las bajo el sistema de Protección Uniforme no tienen medición inicial y su evaluación parte después del primer período de crecimiento.

Los resultados de incrementos en altura por tratamiento (fertilización y distanciamiento a la protección), fueron sometidos a pruebas de Análisis de Varianza y comparaciones múltiples de medias (Prueba de Scheffe).

\section{Características de la Plantación}

La plantación fue realizada a fines de invierno de 1993, con planta 1:0, con escasa selección en vivero. Un alto porcentaje de estas plantas venía desde el vivero con un problema de hongos (presentaban necrosis en el tallo). Por ello fue necesario replantar el año siguiente en un $20 \%$ la faja de $45 \mathrm{~m}$ y un $10 \%$ la faja de $60 \mathrm{~m}$. El análisis estadistico no considero ninguna de estas plantas.

La plantación se hizo a distancias de 3 por 3 metros, (1.111 plantas por hectárea).

Se utilizo gel (Alcosorf) para el transporte de las plantas con el fin de evitar la deshidratación.

Las plantas provienen de semilla colectada en el área de Neltume.

El ensayo de fertilización se hizo con abono foliar (Bayfolan) en dosis de $4 \mathrm{cc}$ por litro, además de un adherente. Se realizaron 2 aplicaciones una a principios de diciembre de 1993 y otra un mes más tarde. a principios de enero de 1994. 


\section{RESULTADOS Y DISCUSIÓN}

\section{Altura y Diámetro de Cuello de las Plantas}

Las alturas y diámetros de cuello de las plantas se pueden observar en el Cuadro $\mathrm{N}^{\circ}$ 1. Las mayores alturas y diámetros corresponden a las plantas ubicadas en la faja de $45 \mathrm{~m}$ de ancho. Estos crecimientos resultan especialmente significativos, ya que las plantas utilizadas venian infectadas con un hongo desde el vivero, además los resultados presentados en el cuadro corresponden al crecimiento obtenido en el borde superior de la faja, donde las condiciones son más rigurosa.

Las plantas establecidas bajo el sistema en fajas han debido competir por luz con otras pioneras, muy agresivas que son parte de la sucesión secundaria que se desarrolla en la faja. entre estas especies se puede mencionar a Chusquea culeou, Cirsium vulgare, Budleja globosa, Muehlenbeckia hastulata, etc.

\section{Cuadro $\mathrm{N}^{\circ} 1$}

\section{ALTURA Y DIAMETRO POR TEMPORADA}

\begin{tabular}{|l|c|c|c|}
\hline Método Silvicola & Variable & I'temporada $^{*}$ & $2^{{ }^{*}}$ Temporada \\
\hline \multirow{2}{*}{ Faja 45 m } & $\mathrm{H}(\mathrm{cm})$ & 33,1 & $83,3^{*}$ \\
\cline { 2 - 4 } & $\mathrm{DAC}(\mathrm{mm})$ & 6,8 & 12,3 \\
\hline \multirow{2}{*}{ Faja $60 \mathrm{~m}$} & $\mathrm{H}(\mathrm{cm})$ & 30,5 & 65,6 \\
\hline \multirow{2}{*}{ Protección Uniforme } & $\mathrm{DAC}(\mathrm{mm})$ & 5,0 & $\mathbf{8 , 4}$ \\
\cline { 2 - 4 } & $\mathrm{H}(\mathrm{cm})$ & 36,9 & 65,8 \\
\cline { 2 - 4 } & $\mathrm{DAC}(\mathrm{mm})$ & 6.1 & 9,2 \\
\hline
\end{tabular}

* = Considera parcelas con fertilización foliar durante el primer año.

\section{Crecimientos}

Los mayores crecimientos. tanto en altura como en diámetro, se presentaron en la faja de $45 \mathrm{~m}$ Cuadros $\mathrm{N}^{\mathrm{os}} 2$ y 3 . Estos crecimientos presentan diferencias significativas $(\mathrm{p}=0.05)$.

\section{Cuadro $\mathrm{N}^{\circ} 2$}

INCREMENTOS EN ALTURA EN LA SEGLNDA TEMIPORADA DE CRECIMIENTO (cm)

\begin{tabular}{|l|c|c|c|c|}
\hline Método Silvicola & N & Media & Mediana & CV \\
\hline Faja 45 m & 336 & 41.6 & 47,7 & 54,6 \\
\hline Faja 60 m & 203 & 37.0 & 30.0 & 66,3 \\
\hline Protección Uniforme & 306 & 34.1 & 31.5 & 60,0 \\
\hline
\end{tabular}

Donde : $\quad \mathrm{N}=$ Tamaño de la muestra

CV = Coeficiente de variacion $(\%)$ 
Estos crecimientos son superiores a los determinados por diversos autores, como González (1994), quien obtuvo incrementos de $21,2 \mathrm{~cm}$ anuales para plantas de 3 años y a los obtenidos por Grosse (1988), quién determinó crecimientos de $24 \mathrm{~cm}$ para plantas con un periodo vegetativo y fertilización.

Por otra parte, Cogollor y Vita (1979), en un análisis de los resultados obtenidos en el ex Complejo Panguipulli en plantaciones a campo abierto, obtuvieron crecimientos iniciales, para los 4 primeros años, entre 30 y $38 \mathrm{~cm}$ anuales.

De igual forma Vita (1974)obtuvo crecimientos para rauli, en Frutillar entre 25 y 30 $\mathrm{cm}$ anuales al cuarto año.

Otros ensayos sobre el ritmo de crecimiento de rauli y coihue, obtenidos por Smulders (1988), entregan crecimientos anuales promedios de $21 \mathrm{~cm}$ y máximos de 58 $\mathrm{cm}$, para ensayos ubicados en la costa de Valdivia.

Por su parte Donoso (1978) señala que el crecimiento del rauli durante los primeros años de vida fluctúa entre 36 y $60 \mathrm{~cm}$.

Crecimiento superiores a los mencionados anteriormente son entregados por Taján (1989), al segundo año en condiciones de vivero y para procedencias de más al norte. Los crecimientos obtenidos por este autor fluctúan entre 47 y $72 \mathrm{~cm}$ anuales promedio.

Cuadro $\mathrm{N}^{\circ} 3$

INCREMENTOS EN DIÁMETRO DE CUELLO DURANTE LA SEGUNDA TEMPORADA DE CRECIMIENTO (mm).

\begin{tabular}{|l|c|c|c|c|}
\hline Método Silvicola & $\mathrm{N}$ & Media & Mediana & CV \\
\hline Faja 45 m & 217 & 5,8 & 5,5 & 50,0 \\
\hline Faja $60 \mathrm{~m}$ & 198 & 3,7 & 3,0 & 70,3 \\
\hline Protección Uniforme & 311 & 3.6 & 3,4 & 53,1 \\
\hline
\end{tabular}

El coeficiente de variación. tanto para incrementos en altura y diámetro, es parecido, es decir ambos caracteres tienen aproximadamente igual variación. Esta variación podria considerarse alta e indicaría un crecimiento inicial tipo "serrucho" (plantación con distintas alturas y diámetros), lo que demuestra la necesidad de homogenizar los individuos seleccionados para ser llevados a la plantación.

\section{Crecimientos de Acuerdo a Ubicación de las Plantas en las Fajas}

De acuerdo a los tratamientos realizados en términos de la ubicación de las plantas dentro de las fajas, los mejores crecimientos en la de $45 \mathrm{~m}$ se obtienen en las plantas ubicadas en el borde inferior. en sus primeros 15 metros y los peores se obtienen en el 
borde superior (Cuadro N 4 ). En este sector de la faja existe una mayor exposición al sol, menor humedad relativa del aire y menor temperatura del suelo (Otero et al,1995).

En la faja de $60 \mathrm{~m}$ los mejores crecimientos se dan al centro $(49 \mathrm{~cm})$, aunque se repiten los menores crecimientos en su parte superior $(28 \mathrm{~cm})$ (Cuadro $\left.\mathrm{N}^{\circ} 4\right)$.

\section{Cuadro $\mathrm{N}^{\circ} 4$}

CRECIMIENTOS DE LA PLANTACIÓN DE ACUERDO

A LA UBICACIÓN DE PLANTAS EN LA FAJA (cm)

\begin{tabular}{|l|c|c|c|}
\hline Faja & Borde Inferior & Borde Intermedio & Borde Superior \\
\hline $45 \mathrm{~m}$ & 62 & 52 & 46 \\
\hline $60 \mathrm{~m}$ & 37 & 49 & 28 \\
\hline
\end{tabular}

F calculado significativo al $95 \%$ de confianza

\section{Efectos de la Fertilización}

La fertilización con abono foliar produjo efectos significativos en el crecimiento en altura aumentando la media en $16 \mathrm{~cm}$. lo que equivale a un $39 \%$ de incremento en altura sobre el control. Las plantas fertilizadas crecieron $57,2 \mathrm{~cm}$ y las plantas sin abono 41,6 cm (Cuadro $\mathrm{N}^{\circ} 5$ ).

\section{Cuadro $\mathrm{N}^{\circ} 5$}

ESTADIGRAFOS DEL ENSAYO DE FERTILIZACIÓN (cm)

\begin{tabular}{|l|c|c|}
\hline Tratamiento & Media & CV \\
\hline C/F & 57,2 & 49 \\
\hline S/F & 41.6 & 49,7 \\
\hline
\end{tabular}

$\begin{array}{rll}\text { Donde: } \mathrm{C} / \mathrm{F} & = & \text { Con fertilización } \\ \mathrm{S} / \mathrm{F} & = & \text { Sin fertilización } \\ \mathrm{CV} & = & \text { Coeficiente de variación }\end{array}$

F significativo al $99 \%$ de confianza.

\section{Sobrevivencia}

La sobrevivencia medida en la segunda temporada es un $82 \%$ en la faja de $60 \mathrm{~m}$, de $89 \%$ en la faja de $45 \mathrm{~m}$ y de un $81.6 \%$ bajo el método de Protección Uniforme (Cuadro $\mathrm{N}^{\circ} 6$ ).

Los porcentajes obtenidos son superiores a los determinados por Grosse (1988), quién establece para el rauli un $50 \%$ de sobrevivencia en plantaciones sin cobertura vertical, aunque similares a los resultados obtenidos por el mismo autor $(84 \%$ a $91 \%$ de sobrevivencia) para el caso de protección uniforme. 
González (1993), también determinó muy bajos indices de mortalidad para esta especie, del orden del $9 \%$ en diversos ensayos a campo abierto.

Debido a los problemas de infección con hongos, durante la primera temporada se secó el $36 \%$ de las plantas, de las cuales un $70 \%$ volvió a brotar al segundo año.

\section{Vigor}

La plantación, a pesar de los problemas iniciales, muestra al tercer año buenas condiciones en cuanto a vigor, siendo entre el $81 \%$ y $91 \%$ clasificadas como plantas vigorosas, (vigor 1).

A pesar de las rigurosas condiciones ambientales existentes entre los 800 y 1000 $\mathrm{msnm}$, las plantas no muestran daños por heladas e insolación y en su gran mayoría están en buenas condiciones sanitarias como lo muestra el alto porcentaje de plantas clasificadas como vigorosas.

\section{Forma}

Un porcentaje relativamente bajo de plantas fue clasificada con doble flecha 0 flechas múltiples, entre 2,4 y $19 \%$.

\section{Cuadro No6}

SOBREVIVENCIA, VIGOR Y FORMA EN LAS PLANTACIONES

\begin{tabular}{|l|c|r|r|r|r|r|}
\hline Método Silvicola & Sobrevivencia (\%) & \multicolumn{3}{|c|}{ Vigor (\%) por Categoria } & \multicolumn{2}{c|}{ Forma (\%) por Tipo } \\
\cline { 3 - 7 } & & \multicolumn{1}{|c|}{1} & \multicolumn{1}{c|}{ (\%) } & \multicolumn{1}{c|}{ ( } & 2 & 2 \\
\hline $\begin{array}{l}\text { Protección } \\
\text { Faja 60 m }\end{array}$ & 82,2 & 84 & 14 & 2 & 97,6 & 2,4 \\
\hline $\begin{array}{l}\text { Protección } \\
\text { Faja 45 m }\end{array}$ & 88,9 & 91 & 7 & 2 & 80,9 & 19,1 \\
\hline $\begin{array}{l}\text { Protección } \\
\text { Uniforme }\end{array}$ & 81,6 & 81 & 8,6 & 10,3 & 83,8 & 16,2 \\
\hline
\end{tabular}

$\begin{array}{rlrl}\text { Donde: } & \text { Vigor } 1=\text { Vigorosas. } & \text { Forma Tipo } 1=\text { Una flecha. } \\ \text { Vigor } 2=\text { Buen estado. } & \text { Forma Tipo } 2=\text { Doble flecha } . \\ \text { Vigor } 3=\text { Plantas debilitadas. } & & \end{array}$

\section{CONCLUSIONES}

Los crecimientos obtenidos bajo el método de Protección en Fajas o protección lateral permiten concluir que esté sistema de corta ofrece muy buenas condiciones para el desarrollo inicial de plantaciones de rauli. 
Pareciera ser que los crecimientos iniciales obtenidos baja estas condiciones son mejores que los obtenidos en plantaciones bajo dosel con protección uniforme o a campo abierto, sin protección lateral.

El abono foliar aplicado a estas plantaciones tiene efectos significativos en el crecimiento en altura de las plantas.

Las mejores condiciones al interior de las fajas. con exposición norte, se encuentran en la zona inferior más protegida o en el centro de la faja. En las partes central y superior la protección lateral es menor y por lo tanto los crecimientos son inferiores.

La sobrevivencia en las plantaciones de raulí es alta, incluso cuando la planta sufre ataque de hongos, lo que indica el fuerte vigor de la especie.

El problema de la doble flecha o forma de las plantas es relativamente menor y además perfectamente corregible.

Los resultados confirman el valor e importancia de la especie en la reforestación del bosque nativo y su importante potencial productivo y por lo tanto económico, demostrado en las plantaciones juveniles existentes en el ex Complejo.

Las variables analizadas presentan una alta variación (CV). es decir un crecimiento heterogéneo. que podría ser normalizado con una buena selección de plantas en vivero.

Las plantaciones alcanzaron una altura promedio que les permitiria ser capaces de imponerse frente a la vegetación mas competitiva.

Los resultados de sobrevivencia. comparados con la escasa regeneración natural que existe en este tipo de bosques. evidencian que la repoblación artificial es el sistema más eficiente en la renovación rápida de la masa boscosa.

\section{RECONOCIMIENTOS}

Se agradece a BOMASA S. A.. empresa que se ha caracterizado por un espiritu de innovación y permanente interés por mejorar el manejo del bosque nativo, particularmente por el apoyo para la realización de las plantaciones y el financiamiento de este estudio. En especial al Sr. Roberto Pérez, encargado de silvicultura de la empresa.

Se agradece también al Sr. Tomás Monfil, silvicultor de amplia trayectoria en bosque nativo, por los consejos y apoyo prestado durante la plantación de las fajas.

Al Sr. Pablo Donoso. actualmente profesor de la Universidad Austral, quién participó en el establecimiento de las plantaciones. 


\section{REFERENCIAS BIBLIOGRÁFICAS}

Contreras, A. Otero L Barrales LOjeda L, 1995. Estudio de Crecimiento de una Plantación de Rauli en el Sector de Panguipulli. Instituto Forestal .Valdivia. En Prensa.

Cogollor G y Vita A., 1979. Regeneración en Bosque Nativo de Rauli. Plantaciones y Ensayos de Regeneración. U. de chile Fac. cs. Forestales. Santiago.

Danby, N., 1991. Nothofagus in Wales. Journal of Forestry Vol LXXXV N 2.

Donoso C., 1989. Regeneración y Crecimiento del Tipo Forestal Siempreverde Costero y Andino Tras Distintos Tratamientos Silviculturales. Rev.Bosque Vol $10 \mathrm{~N} I$

Donoso P.Monfil T.Otero,LBarrales L, 1993. Estudio de Crecimiento de Plantaciones y Renovales Manejados de Especies Nativas en el Área Andina de la Provincia de Cautin y Valdivia. Instituto Forestal Valdivia. Vol 7 N 2.

Donoso C., 1978. La Silvicultura de Nothofagus en Chile. Dep. Silvicultura U. de California.USA.

González, M., 1993. Estado de Efectos de Diferentes Regimenes de Acondicionamiento de Plantas de Rauli (Nothofagus alpina), 1 - 0 Raiz Desnuda. Tesis U. Austral

Grosse H., 1988. Crecimiento de Plantaciones de Rauli y Roble Bajo Dosel en Dependencia del Grado de Luminosidad y Fertilización. Instituto Forestal. Rev. Ciencia e Investigación Forestal Vol $2 \mathrm{~N} 5$.

Ocaña-Vidal, J., 1992. Ordenación de Bosques Naturales Mediante Franjas Protectoras. Unasylva 169. Vol43.

Otero, LContreras A. Barrales, L, 1994. Efectos Ambientales de las Cortas de Protección en Bosque Nativo. Seminario Medio Ambiente y Biodiversidad. Instituto Forestal . Santiago.

Otero, L Contreras, A. Barrales, L, 1994. Efectos Ambientales de Diferentes Tipos de Corta en Bosque Nativo. El Caso de las Cortas de Protección en Fajas. Ciencia e Investigación Forestal. Vol $8 \mathrm{~N}^{\circ} 1$. Instituto Forestal. Santiago.

Smulders A., 1998. Estudio del Ritmo de Crecimiento de Nothofagua alpina, Nothofagus dombeyi y Persea lingue Durante los primeros Años de Vida. Tesis Fac. Cs Forestales U. Austral.

Taján P., 1989. Crecimiento y Resistencia a la Sequia de Ocho Procedencias de Rauli Durante el Segundo Periodo Vegetativo en Valdivia. Tesis Fac. Cs. Forestales. U. Austral.

Tuley, G., 1979. Nothofagus in Britain.Forestry Commission. Forest Record.England

Vita A., 1974 Algunos Antecedentes para la Silvicultura del Raulí.Fac. Cs. Forestales. Bol Téc. N 18. 